\title{
An introduction to the memristor - a valuable circuit element in bioelectricity and bioimpedance
}

\author{
Gorm K. Johnsen ${ }^{1,2}$ \\ 1. Institute of Energy Technology, 2007 Kjeller, Norway \\ 2. Department of Physics, University of Oslo, 0316 Oslo, Norway \\ 3. E-mail any correspondence to: g.k.johnsen@fys.uio.no
}

\begin{abstract}
The memristor (short for memory resistor) is a yet quite unknown circuit element, though equally fundamental as resistors, capacitors, and coils. It was predicted from theory arguments nearly 40 years ago, but not realized as a physical component until recently. The memristor shows many interesting features when describing electrical phenomena, especially at small (molecular or cellular) scales and can in particular be useful for bioimpedance and bioelectricity modeling. It can also give us a richer and much improved conceptual understanding of many such phenomena. Up until today the tools available for circuit modeling have been restricted to the three circuit elements (RLC) as well as the widely used constant phase element (CPE). However, as one element has been missing in our modeling toolbox, many bioelectrical phenomena may have been described incompletely as they are indeed memristive. Such memristive behavior is not possible to capture within a traditional RLC framework. In this paper we will introduce the memristor and look at bioelectrical memristive phenomena. The goal is to explain the new memristor's properties in a simple manner as well as to highlight its importance and relevance. We conclude that memristors must be included as a readily used building block for bioimpedance and bioelectrical data analysis and modeling.
\end{abstract}

Keywords: Memristor, bioimpedance, bioelectricity, new circuit element, electrical memory.

\section{Introduction}

In every introductory course in electromagnetism or circuit theory one learns that there are three fundamental circuit elements, namely the capacitor (first realized in 1745), the resistor (1828) and the coil (1831). When Maxwell announced his famous and extremely powerful equations some decades later, it became clear these three elements were direct consequences of this theory that unifies electricity and magnetism. However, in 1971 a young circuit theorist, Leon Chua argued from theoretical grounds that there should be also a fourth circuit element that was equally fundamental as the other three [1]. He gave this element the name memristor since it, under certain conditions, acts as a resistor with memory, i.e. the resistance is dependent on the physical history. In the years following Chua's work, the memristor concept was left quite alone until the birth of nanotechnology some years ago when a group at the Hewlett-Packard (HP) lab managed to construct a physical component acting as a memristor $[2,3]$. An image of this memristor is given in Figure 1.

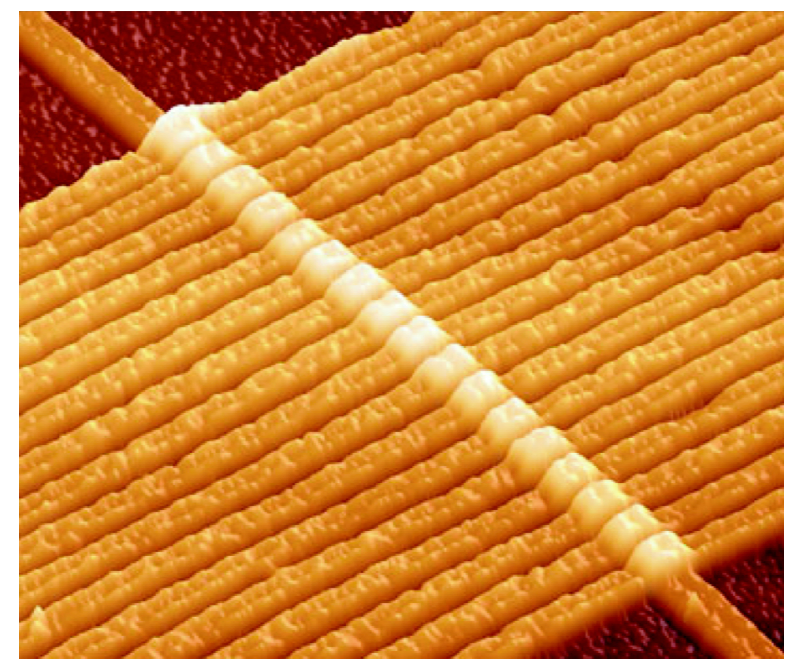

Fig. 1: The first realized memristor, produced in the HP lab. Seventeen nano-memristors are shown in parallel. (This image was produced by R. Stanley Williams of the HP lab and it is reproduced with permission from R. Stanley Williams.)

"So what?" you might think. An additional circuit element may not sound that spectacular, but imagine that you have been living for nearly 40 years with socks, shoes, and sweaters, but no trouser and then suddenly get a pair. In the beginning it may be hard to see the potential of a newly introduced fourth element and difficult to use properly, often mixing it up with the other three; however, if it turns out to be more suitable to certain tasks, who knows how useful it might be in the end?

In this paper we will argue that the memristor is a necessary and useful building block in circuit theory and therefore also necessary and useful in modeling bioelectrical phenomena. We will first explain and show examples of areas where the memristor already has been used. Furthermore, we will look into other electrical phenomena that are likely better described by the "new" memristor element than the traditional RLCs (or the constant phase element (CPE) for that matter). The important lesson to be pulled out of this paper is that, from now on any current responding to an applied (harmonic) voltage should be studied and interpreted with the memristor element (or other memory circuit elements) ready in the modeling toolbox. The different topics in this review-like paper are covered in such a way that it should be possible to jump to those sections of most interest when the basic memristor theory is well understood. 


\section{Historical background of the memristor}

In this section we will consider briefly how fundamental the memristor actually is and how easy it can be justified from a simple symmetry diagram. The fact that memristors were hidden within Maxwell's equations for more than a century of extensive use is actually a bit of a mystery, but one suggestion is that Maxwell's equations originally were not formulated in the form we know today [3]. Another is that memristive systems, as we will see, are usually very small in size and therefore were not able to be identified until the recent birth of nanoelectronics and the corresponding study of charge transport in solid-state and biological systems.

In the late 1960's Leon Chua studied the mathematical fundamentals of circuit theory. One subject he had a certain interest in was the possible relationship between pairs of circuit variables. From a theoretical point of view the three basic circuit elements - resistor, capacitor, and coil - are defined by a relationship between two of the four axiomatic circuit variables: the voltage, v; the current, $\mathrm{i}$; the charge, $\mathrm{q}$; and the flux $\varphi$. These four variables lead to six possible combinations (see Appendix for more details). Chua noted that only five of these combinations had led to established relationships [4]. Two of the five realizations are the definition of current $\mathrm{i}=\mathrm{dq} / \mathrm{dt}$ and Faradays law of induction

$$
\varphi=\int_{-\infty}^{t} v(\tau) d \tau
$$

The other three are the definitions of the well-known basic circuit elements which are the capacitor $(\mathrm{C}=d \mathrm{q} / d \mathrm{v})$, the resistor $(\mathrm{R}=\mathrm{dv} / \mathrm{di})$, and the coil $(\mathrm{L}=\mathrm{d} \varphi / \mathrm{di})$. This means that one relationship remained undefined, namely the correlation between electric charge, q, and magnetic flux, $\varphi$, which we can be seen from the symmetry diagram in Figure 2.

Chua postulated an additional circuit element defined by the relation between charge and flux should exist, from both a logical point of view and for the sake of completeness [1]. Chua called this circuit element a memristor (short for memory resistor) based on the properties he found the element to have. Put simply, it was an element that changed its resistance depending on how much charge flowed through it. Since Chua was not able to realize the memristor himself at that time, it remained a puzzle for almost four decades. Nonetheless, Chua showed that the memristor cannot be replaced by any combination of the other three passive circuit elements, no matter how nonlinear these elements are. Still, as we will see later in this paper, the memristor will degenerate to an ordinary resistor under certain conditions.

Figure 3 shows the electronic symbol representation of a memristor element. From the symmetry diagram in Figure 2, we see how the memristor finds its natural place in the circuit theory along with the five other combinations of pairs of circuit variables. With this simple diagram in front of us, one might wonder why it took so long until the memristor was identified.

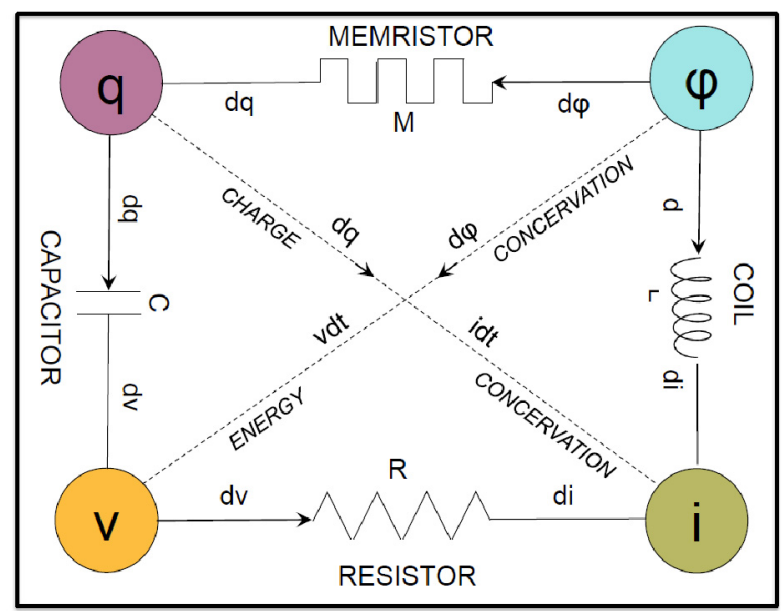

Fig. 2: Symmetry diagram showing the 6 distinct possible realizations based on the four circuit variables. Courtesy of $\mathrm{C}$. Lütken [4].

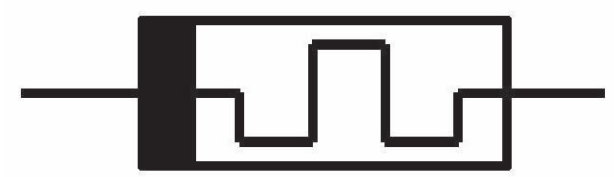

Fig. 3: Symbol representing a memristor in an electric circuit.

\section{Definition of the memristor}

The relationship between flux and charge defines memristance as

$$
M=\frac{d \varphi}{d q}
$$

This concept was generalized to memristive systems by Chua in 1976 [5] and can be written as

$$
\begin{aligned}
& v=R(x, t) i \\
& \frac{d x}{d t}=f(x, t),
\end{aligned}
$$

which looks like a nonlinear version of Ohm's law. One important aspect to keep in mind about the memristor is its dependence on the "state" variable $\mathrm{x}$. The state variable describes how the system "looks" inside. It is time dependent, a fact that makes the memristor a non-linear element distinct from the (linear) resistor. This timedependence of the state variable provides the "memory" of the system. In biological systems, the state variable is often proportional to the electrical charge (although not always). Thus the resistance will vary with the amount of charge passed through the element and in general not be a constant. 


\section{An illustrative example: The first memristor}

Almost 40 years after Chua had postulated the memristor to exist, it was described for the first time as a physical system by a group of researchers at the HP lab in California, USA [2]. The nano-sized memristor they developed consisted of a thin titanium dioxide, $\mathrm{TiO}_{2}$, layer that was sandwiched between two platinum electrodes and is shown in Figure 1. As this particular memristor model is as simple as it is illustrative, we will investigate it in what follows and use it to highlight what is typical for memristors. This memristor model is also very similar to the memristance that can be found in electrical measurements of the skin as will be seen later in this paper.

An illustration of the HP memristor is shown in Figure 4. This way of illustrating memristors, as a component separated in two parts, is very useful to understanding the basic principles. The memristor consists of one part that conducts current well (the doped part), and one part that is a much poorer electrical conductor (the undoped part). The total resistance - or strictly speaking, the memristance - of this two-component system is the sum of the doped and the undoped part. What makes this system a memristor and not a resistor is the presence of a dynamic state variable defining the boundary between the two parts with high and low conductance. The state variable ( $\mathrm{w}$ in this particular case) describes how the memristance of the element evolves with time. For this particular HP memristor the state variable $\mathrm{w}$ is proportional to the electric charge $\mathrm{q}$.

When current is passing through this HP memristor, the boundary between the high and low conductive states is shifted either to the right or to the left with time. It can be thought that the charge carriers push this boundary back and forth. This means the total memristance of the system is either reduced or increased depending on in which direction the charge carriers flow. This is exactly what is needed for a memristor. The memristance of this system can be written as:

$$
M(q)=R_{1}+R_{2}
$$

Here, $R_{1}$ and $R_{2}$ are the low and high resistive part of the memristor. This expression can furthermore be written on a form that is illustrative for memristors [2]

$$
M(q)=R_{1}+R_{2}=R_{O F F}\left(1-\frac{k R_{O N}}{D^{2}} q(t)\right)
$$

where $R_{\mathrm{OFF}}$ and $\mathrm{R}_{\mathrm{ON}}$ are the highest and lowest possible resistance of the memristor, respectively, $\mathrm{k}$ is a constant, $\mathrm{D}$ is the length of the memristor in the direction of the charge flow, and $q(\mathrm{t})$ is the electric charge passing through the memristor as a function of time. We note the following four things:

1. The dependence of the electric charge shows that this system is not resistive, but instead memristive.
2. The memristive contribution to the electrical behavior of the system blows up when the device itself becomes very small.

3. The expression of the memristance does not explicitly involve magnetic flux, although the memristor is defined as a relation between flux and electric charge.

4. The physical unit of memristance is expressed in ohms.

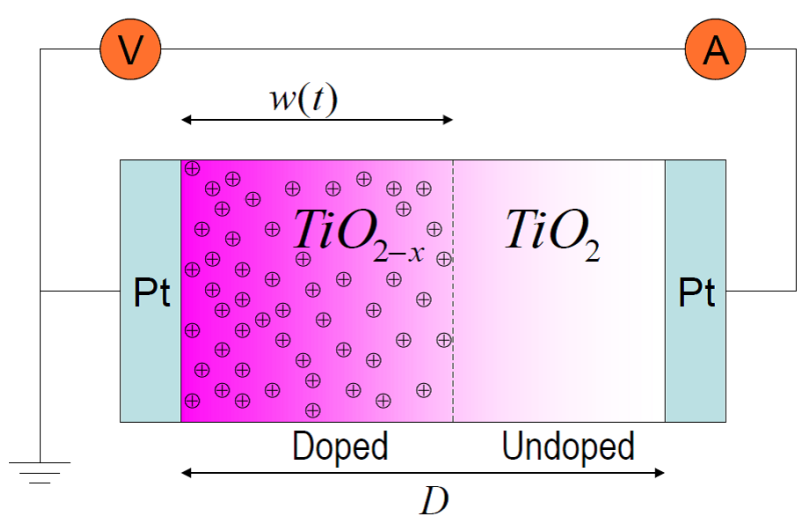

Fig. 4: Cross-section of the first $\mathrm{HP} \mathrm{TiO}_{2}$-memristor consisting of a high conductive (doped) and a low conductive (undoped) part placed in between two platinum electrodes. The boundary between the two parts is dynamic and is moved back and forth by the passing charge carriers. The parameter $\mathrm{w}(\mathrm{t})$ is a mathematic variable that describes the position of this boundary [4]. Courtesy of C. Lütken.

The apparent absence of flux can be explained by using Faraday's law of induction rewriting the definition of the memristor as

$$
M=\frac{d \varphi}{d q}=\frac{v d t}{i d t}=\frac{v}{i}
$$

This just looks like ordinary resistance (but the charge dependence ensures that it is really not), and hence it works as an illustration that memristance in some cases degenerates to ordinary resistance. This happens, for example, if $\mathrm{M}(\mathrm{q})$ is constant with time. Thus, it seems clear that there is no need to introduce memristors in linear circuit theory. Memristors are nonlinear.

When describing the first memristor, the HP researchers made use of this fact of nonlinearity. Instead of searching among magnetic systems they started investigating small electrical devices (when D is small) where there was a nonlinear relation between the integral of the current and the integral of the charge so that the $M(q)$ becomes nonlinear [2,7]. It has been speculated that the reason such a long time passed from when the memristor first was postulated (1971) until it was described as a real physical device (2008) was because people were searching among systems involving magnetic flux, which made the chase much more difficult [7].

So what does this mean? Memristors are obviously nonlinear systems of small size. This is something that is found in many biological systems, prompting the need for further study. Especially those systems that exhibit a 
dynamic behavior of the resistance with time may be promising candidates for being memristive rather than any other.

\section{A useful water analogy}

Because the memristor element might still be relatively unknown in our field, in this section we will describe its basic properties via a simple analogy with a water pipe. Many phenomena in electrical circuit theory can be explained by using an analogy where water flows in pipe systems. The same is true for memristors. The example we expound here is similar to what was done in the early work of Lütken et al. [4,8]. The principle outlined below is also very well suited to illustrate the main content of Strukhov et al. [2], who described the first memristor, and Johnsen et al. [9], wherein the electrical properties of skin were shown to be memristive due to electro-osmotic transport of fluid in the sweat capillaries.

In the water pipe analogy, ordinary resistors can usually be thought of as the pipe with water flowing through it. The wider the pipe the lower the resistance felt by the water. The memristor, which has some similarities to the resistor, must therefore be some kind of a modified water pipe in this context. If we jump back to equation 3, we see that the memristor consists of two parts, one with high resistance, the other with low resistance, and so a twocomponent pipe as shown in Figure 5 is appropriate.

The thin and thick tubes in the model are the two main parts in the memristor, offering high and low resistance to the water, respectively. The two fat tubes on either end of this system are the platinum electrodes in the HP memristor and offer no resistance to the flow of water. For this system to be a memristor, there must be a dynamical change of the memristance with time. This is where the piston in Figure 5 comes in. The piston is located at the boundary between the high resistance part (thin tube) and the low resistance part (larger tube), and plays the same role as the w parameter in the HP model in Figure 4. Depending on which direction the water flows in the memristor, the piston is pushed either to the right or to the left, changing the total memristance of the system from high to low or vice versa.

\section{Basic memristor properties}

Listed below are some of the most characteristic and important features for memristors:

- An ac element, not dc

- No storage of energy

- Two-point terminal circuit element

- Pinched hysteresis loop in the i-v plane

- Positive or negative differential resistance

- Nonlinear q- $\varphi$ curve

- Low-frequency property and frequency-dependent memristance; and

- Typically only apparent at small scales.
Some of the bullet points in this list are typical fingerprints of memristors and should be searched for when analyzing data from electrical measurements. Others are more generic properties for memristors that distinguishes them from other building blocks in circuit theory. We shall in the following see how these properties can be explained relatively simply with support from the water analogy.

We have seen how the memristance of the memristor changes with time when an alternating current is passing through the device, indicating that it is an ac device. In general memristance is not visible for dc signals. Still, the memristor will conduct dc current, but now behave as a time-varying resistor and not as a proper memristor. If we go back to our water analogy of the memristor, shown in figure 5, a dc (water) current will push the piston only in one direction all the time, making the piston reach one of the "ends" of the system and consequently the memristance then saturates. This means the apparent resistance (the memristance) of the pipe system does not alternate dynamically with time, which is required for the device to behave as a memristor. Instead, the memristance is timevarying during the (short) initial period as the piston is being moved and thereafter the memristor degenerates to an ordinary resistor as the piston reaches either side of the water pipe system.

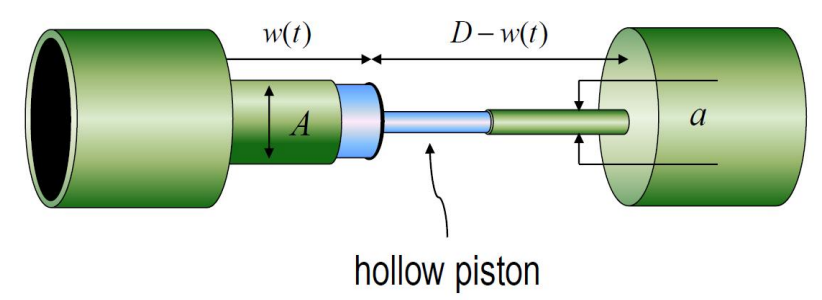

Fig. 5: Pipe model of a memristor. Here, the thick tube, A represents the high conductive part of the memristor, whereas the thin tube, a represents the high resistive part. Depending on the direction of the water flow, the piston is pushed either to the left or to the right. Hence, the net resistance of the memristor is altered when water is flowing through it. This model has also earlier been presented in [4]. Courtesy of C. Lütken.

Once the voltage over the memristor is switched off, the dynamic motion of the boundary between the two parts stop and the resistance value of the memristor is preserved - or, put colloquially, "remembered." This memory of the device is kept until another voltage is applied to the system, inducing charge transport. How long this memory may be preserved, depends on how the system looks at its "bottom level." For solid-state systems such as the HP memristor, memory may last a very long time $[2,10]$. For biological systems it may degenerate more rapidly when the driving voltage is turned off as the state of the system is influenced by a number of other factors such as diffusion [9]. Even if the resistance state of the memristor is preserved, the memristor does not involve any storage of electric or magnetic energy.

A memristor, which is a two-terminal circuit element, will provide hysteresis loops in an $\mathrm{i}-\mathrm{v}$ plot when subject to an alternating voltage signal. An example of an ideal model 
of a memristor is shown in Figure 6. The hysteresis loops are very valuable when memristive systems are to be identified, and the loops normally run through the origin in an $\mathrm{i}-\mathrm{v}$ plot. This behavior can be seen to arise from that described in equation (3). When $v=0$, then $i=0$ and vice versa. This fact further underlines that the memristor is not an energy storage device. The hysteresis loops are formed since the current through the memristor does not vary linearly with the applied voltage, unlike a resistor that follows Ohm's law.

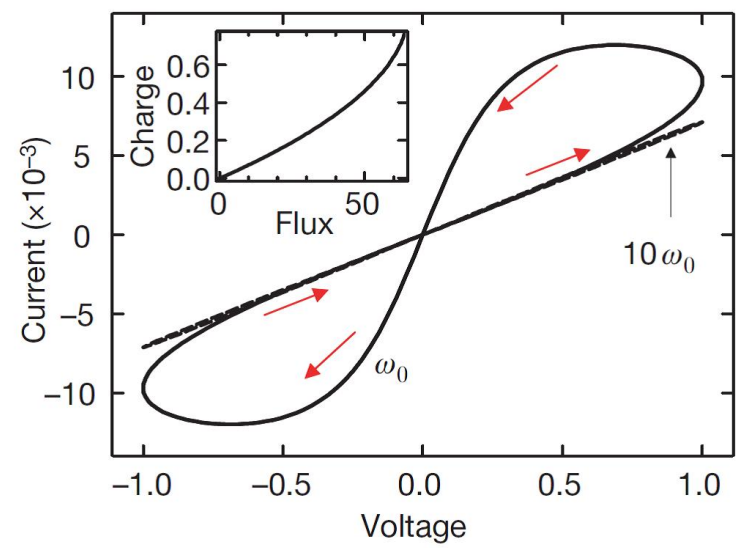

Fig. 6: Typical behavior of memristors. The memristance of the memristor depends on the amount of electric charge that has passed through the device. Consequently, the current is nonlinear with the applied voltage, resulting in hysteresis loops rather than straight lines. If the signal frequency, $\omega$, is sufficiently high, the memristance of the memristor has too little time to respond to the passing charge, resulting in the collapse of the hysteresis loops to straight lines. The inset figure shows that memristors require nonlinear $q$ - $\varphi$-plots. Reprinted from Nature with permission [2].

We can justify this to ourselves by remembering that the boundary, w, between the high conductive state and the low conductive state of the device is moved by the charge carriers as current is passed the memristor (see Figure 4). If the charge carriers push the boundary to one side the instantaneous resistance of the two-component device is reducing with time. This means that current is increasing at an increasing rate compared to the voltage. This is not linear and the i-v curve curves upward, as seen in the upper right corner in Figure 6. Even after the voltage starts to decrease, the current increases for a short while since the charge carriers still push the boundary towards a low conductive state (i.e. to the right in figure 4). This feedback, which can be either positive or negative depending on the direction of motion the charge carriers, is typical for memristors and differs from what we are familiar with through Ohm's law, where $i=\mathrm{v} / \mathrm{r}$.

From the inset figure of Figure 6 we see how a memristor gives a nonlinear curve when the flux, $\varphi$ is plotted versus the charge, q. This nonlinear behavior is required for a system to be memristive. Hence, any hysteresis detection of current versus voltage should be tested to see if charge varies nonlinearly with the flux, an indication of the system might being memristive.

Memristors and memristive systems will typically be frequency dependent, meaning that the measured memristance of the system will depend on the frequency, $\omega$, of the applied signal. We can grasp this better by jumping back to the water pipe analogy in Figure 5. When the applied frequency increases, there will be less time for the piston to move to the right or left, since now the flow of water changes direction faster and faster. Eventually, when the frequency is sufficiently high, the piston will be left practically at rest with only minimal vibrations back and forth. Consequently, there is no longer a dynamic change of the total resistance of the water pipe. Hence, the hysteresis loops are collapsing to a straight line and the degree of collapse increases with frequency. Consequently, the memristor acts as an ordinary resistor. The frequency value responsible for such a collapse of the hysteresis curves depends on the internal states of each system and will therefore generally not be the same for different types of memristors.

From equation (5) we see how the memristive term blows up as the typical size, D, of the system is reduced. For example, if the size changes from $\mu \mathrm{m}$ to nm scale, the memristive contribution is increased by a factor of $10^{6}$. One may therefore expect memristive effects to become more significant as studies on smaller and smaller electrical systems are carried out. Fundamental electrical mechanisms behind many bioelectrical phenomena may therefore involve significant memristive effects. Chua mentioned biophysics as a field where "mistaken nonlinear identities" are present in the literature and emphasized the need to sort out some of the misunderstandings by including memristive systems in circuit models $[1,11]$. Certainly, understanding small scale, nonlinear electrical phenomena will become more and more important as time goes by.

Should the reader need it, a further detailed description about the memristor's properties in an electric circuit can be found in Yoglekar et al. [12].

\section{Memristors and bioimpedance}

Within the field of bioimpedance and bioelectricity the memristor may be a valuable tool for circuit modeling and fundamental physical insight. There are a many observed hysteretic, anomalous or nonlinear i-v characteristics in nature [13-15] that may potentially fit better in a memristive framework than in any other. Typically, as we have seen, memristive fingerprints are visible at systems where the scales of the characteristic electrical processes are small (for instance biological systems at cell levels). The scales where memristive effects have been reported so far $[2,3,9]$ are in the same range where the electrical properties of biological systems such as cells and tissue constituents start to emerge from the underlying (molecular) physics. We should keep in mind that some of the underlying physics might be described more correctly in terms of the memristor rather than the traditional RLC or CPE.

Since the memristor has been not yet received the attention of the other fundamental components of circuits the resistor, the capacitor and the coil - there exists a significant possibility that systems which indeed are 
memristive may have been described incompletely, potentially as systems showing anomalous i-v characteristics. This has been discussed at length by Chua $[11,17]$.

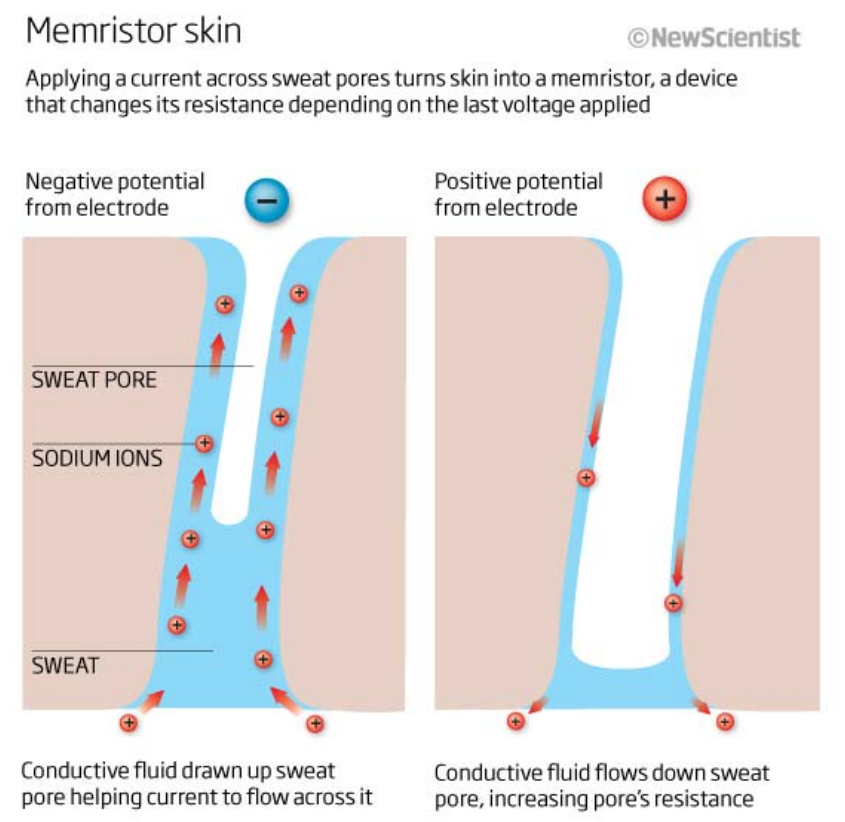

Fig. 7: Cross-sectional view of a sweat pore in the skin. From New Scientist with permission [16], (C) 2011 Reed Business Information Ltd, England. All rights reserved. Distributed by Tribune Media Services).

In the following sections, we will go through some bioelectrical phenomena that are good candidates for being memristive. Within the field of bioimpedance and bioelectricity, circuit modeling plays a very important role and helps us to understand tissue properties [18,19]. A new circuit element, which also captures nonlinear phenomena (e.g. the hysteresis loops) is very welcome in such a field. Now we are relatively certain that we have the full set of fundamental circuit elements at our disposal when we want to describe phenomena observed by our measurements.

\section{Skin conductance and ionic channels}

Skin conductance measurements performed at low frequencies have revealed that the missing memristor has been at our nose tip the whole time. Our skin behaves as a memristor due to the dominance of the sweat ducts on the skin conductance at sufficiently low frequencies [9,20]. An illustration of such a sweat duct, partly filled with liquid, is shown in Figure 7. When a low-frequency signal is applied to the skin and its many sweat ducts, the degree of liquid filling will depend on the amount of charge that passes through the ducts. When a negative voltage signal is applied the liquid level rises through the electro-osmosis process [20]. As the duct becomes more filled with sweat liquid, the conductance increases since sweat conducts current very well [21-23]. If the polarity of the signal is positive, the liquid level is driven downwards in the duct, resulting in a decrease of the overall skin conductance. So when measuring skin conductance at low frequencies, we observe that it changes dynamically with time when current is passing through the skin. The actual dependence is based on how much charge has passed through the duct since each charge carrier pushes the liquid along with its motion by electro-osmotic forces. It turns out each of us are all wrapped up in a biological memristor.

The equivalent electrical model of the human skin may therefore involve the memristor as suggested in Figure 8 when doing impedance measurements. The new thing in this manner is that the traditional resistor for sweat conductance is substituted with the memristor, as highlighted in red in Figure 8.

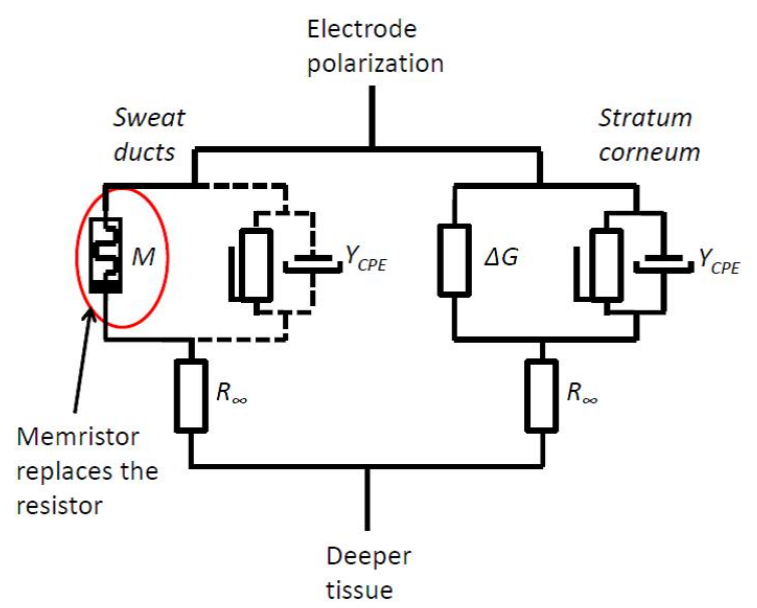

Fig. 8: Equivalent electrical circuit model of the skin, now also with the memristor to capture the sweat duct properties.

Furthermore, this skin model can be simplified based on our detailed knowledge of the constituents in the circuit. For example, the electrical properties of the sweat ducts are dominated by the liquid, which is memristive in small capillaries and not influenced by capacitive effects [24]. This is why the parallel branch involving the CPE is drawn with dotted lines in Figure 8 . Note that when the applied frequencies are sufficiently high, the memristor turns into a resistor as we have seen earlier, and the skin model returns to its traditional form [18]. Note that the memristor is capable of conducting dc currents, and so the model in Figure 8 covers also that mechanism.

More generally, many kinds of biological capillaries and duct systems will be able to behave memristively if they are partially filled with some kind of conductive liquid. Such channels are very frequent in biological systems making them promising and interesting candidates for being memristive in nature. This was in fact suggested already by Chua in one of his early papers [6].

\section{Memristive cell action potential}

The fundamental cell electrical unit is the action potential whose origin is located across the cell membrane. When potassium and sodium ions are transported across the membrane, a potential is released, making it possible for cells to be electrically active. First described by Hodgkin and Huxley in the 1950s, this potential is in many ways the fundamental building block of bioelectricity [25]. 


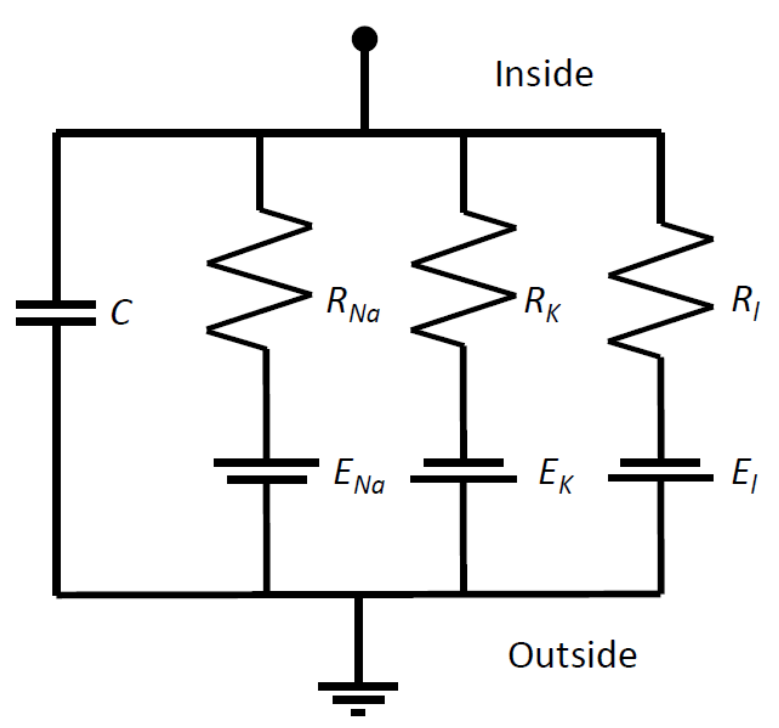

Fig. 9: Equivalent electrical circuit of the Hodgkin-Huxley nerve membrane model in its original form. Here, "inside" and "outside" denote the interior and exterior of the cell with the membrane separating these two parts. Furthermore, $\mathrm{C}$ is the membrane capacitance, $R_{\mathrm{Na}}$ and $\mathrm{R}_{\mathrm{K}}$ describe the sodium and potassium time-varying conductivities, respectively, $R_{1}$ describes the leakage resistance, $\mathrm{E}_{\mathrm{Na}}, \mathrm{E}_{\mathrm{K}}$, and $\mathrm{E}_{1}$ are the reverse ion channel potentials.

The equivalent circuit of the Hodgkin-Huxley model is shown in Figure 9. The model describes charge transport across a nerve cell membrane by introducing specific channels for potassium and sodium ions, a leakage channel, a capacitor describing the properties of the membrane itself. For more rigorous introductions to the Hodgkin-Huxley theory we recommend consulting Johnston \& Wu (1994) and Dayan \& Abbott (2001) [26,27].

The model of Hodgkin and Huxley today stands as one of the greatest achievements in neuroscience and bioelectricity, and eventually the two men responsible received the Nobel Prize in Medicine for their work. At the time when Hodgkin and Huxley made their model, they naturally did not know about the memristor, but they showed that the action potentials in neurons will have a history-dependent conductance of the membrane channels. Motivated by this fact Chua showed, some 20 years later, that the Hodgkin-Huxley model is essentially memristive [6]. The nerve cell membrane, as suggested by Hodgkin and Huxley, was modeled by means of three separate channels where ions could pass. Apart from a leaking channel, it consisted of channels for sodium and potassium transport through the membrane. The conductance of these two channels depends on time and voltage. This fact is crucial to making their behavior memristive rather than strictly resistive. An equivalent electrical model of the nerve cell membrane, as revised by Chua to contain the memristor is shown in Figure $10[6,17]$. This model is conceptually distinct from the traditional Hodgkin-Huxley nerve membrane model because it now contains the proper circuit element to capture the history dependence in the membrane channel conductivities.

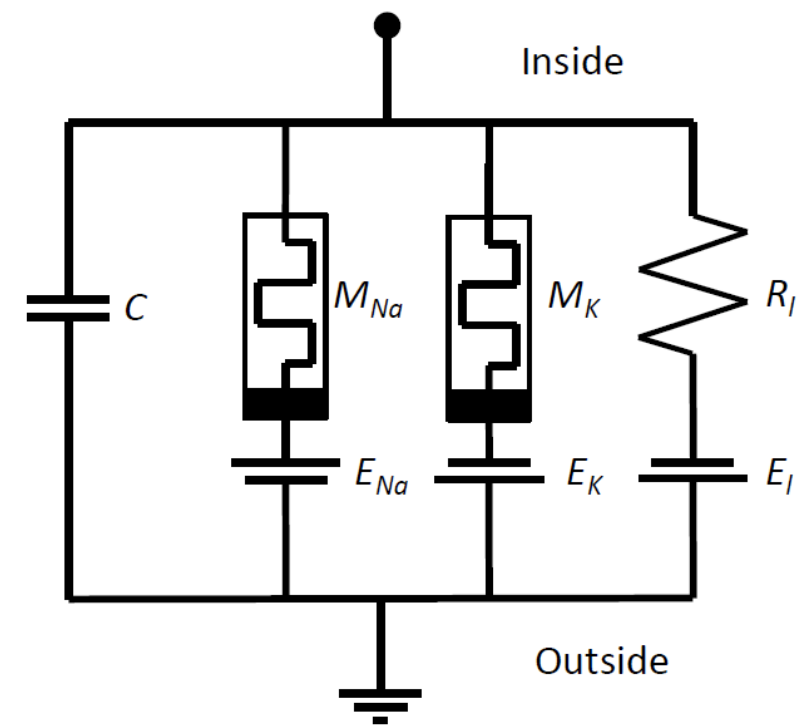

Fig. 10: Memristive Hodgkin-Huxley model. Note that the timevarying resistances in figure 9 are replaced by memristors.

As the Hodgkin-Huxley model is also the starting point for simulations of nerve cell activity, there is now a hope that the "new" memristive model will significantly push neuroscience forward, though this effect remains to be seen. The memristive model of the cell action potential was presented in a non-biological journal and remained unknown until recently as memristors have begun to garner more and more attention. A fine introduction to the how neural electrical activity is modeled is given by Einevoll [28] and is suggested for further reading.

\section{Neural networks and memory}

From the new memristive descriptions of the electrical unit in the cells, more memristive models of bioelectricity and neuroscience have emerged. For instance, a model of a memristive electronic circuit aiming to capture the processes of learning and associative memory of neural networks has been proposed by Pershin and Di Ventra [29]. Also it is believed that memristors could mimic synaptic behavior and mechanisms of memory $[17,30]$ and cellular memory in the human brain [31].

\section{Identifying memristance in your data}

Now that we have looked through some of the most relevant topics for present and future memristive behavior the next step is to actually start to search for more. Therefore, let us quickly refresh how to detect memristive effects present in experimental data:

- Look for pinched hysteresis loops in i-v plots.

- Alternatively, check if current versus time curves, following a harmonic applied potential, are "leaning" either to the right or to the left. This may indicate there is a nonlinear resistive behavior of the system. 
- Look for nonlinear q- $\varphi$ curves (i.e. integrate up current and voltage and plot them against one another).

The first two bullet points should always be tested against the final one as this is the actual definition of memristors.

\section{Memory circuit elements in general - memory capacitors and inductors}

In this section we will briefly mention the more general concept of memory circuit elements, not strictly the memristor. A thorough detailing of memory circuit elements is a too sizeable a task to fully go into here. Instead, we will only mention them briefly and provide some literature for further reading [10,33].

In 2009 Di Ventra et al. extended the concept of memory circuit elements by introducing memcapacitors and meminductors [32]. Just like the memristor acts as a resistor with memory, memcapacitors and meminductors act like capacitors and coils (inductors) with some memory dependent on their physical history. These two additional memory devices share many of the properties with the memristive systems, but with an important difference; they are capable of storing energy just like ordinary capacitors and coils. It is believed that these elements will be very useful as scientific investigations proceed towards smaller and smaller electrical circuits. In particular the memcapacitor is believed to be useful within bioelectricity and neuroscience [10], potentially mimicking the widely used CPE [9].

\section{Memristors and the future}

It is not so easy at this rather early stage to say much more about the practical relevance of the new circuit elements to bioelectricity and bioimpedance. From a theoretical point of view, however, the importance of the memristor is indisputable as its fills a gap in the theory. Because there are many memristive "fingerprints" within bioelectricity that have not yet been properly investigated at present, it is quite likely the memristor will turn out to be useful. We know that the literature reports anomalous phenomena [11], which have heretofore been modeled and explained with an insufficient and incomplete theory. This fact alone is enough to initiate a revision of many old results, bearing the memristive toolkit in mind. That memristive effects are typically present at small scales in ionic membrane systems makes nano-biology very interesting in this context.

\section{Conclusion}

In this tutorial article, we have shown that memory circuit elements, and the memristor in particular, have a natural place in circuit theory. And even if it only came to fruition in 2008, memristive experimental observations actually date far back in time [34], they were just not properly identified. Consequently, the memristor is from now on as natural as any other circuit elements when electrical phenomena are to be explained and understood. In many cases the memristor concept also has the potential to give us a richer and more conceptually correct understanding of nature as it opens a neglected field in bioelectricity and bioimpedance. Though the memristor is fundamental, it still rather unknown, meaning that it is very likely that it has been very underutilized up until today. Therefore, many systems that earlier were thought to be anomalous, may actually be memristive, or memcapacitive or meminductive for that matter. Still, there remains much work to be done to implement these memory elements properly in our field.

\section{Acknowledgements}

The author is indebted to Carsten Lütken for useful discussions and input, as well as to R. Stanley Williams and New Scientist for granting permissions for reuse of graphical material.

\section{Appendix}

This section simply aims to quickly see how the four circuit variables $\mathrm{i}, \mathrm{v}, \mathrm{q}$, and $\varphi$ lead to the six different realizations, including also the memristor. From the four variables, in principle $16\left(=4^{2}\right)$ possible realizations are possible.

However, as four of these are degenerate $(\mathrm{dv} / \mathrm{dv}, \mathrm{di} / \mathrm{di}$ and so on), only 12 are left. Furthermore, due to symmetry grounds, $12 / 2=6$ distinct realizations are left, which are shown in the diagram in figure 2 . This symmetry simply reflects that permutations of two variables (i.e. $\mathrm{dv} / \mathrm{di}=\mathrm{R}$ and $\mathrm{di} / \mathrm{dv}=1 / \mathrm{R}$ ) contain the same physics. A more rigorous review on how the four circuit variables can be deduced from Maxwell's equations can be found in Kavehei et al. [35].

\section{References}

1. Chua LO. Memristor - The missing circuit element. IEEE Trans. Circuit Theory. 1971;18;507-19. http://dx.doi.org/10.1109/TCT.1971.1083337

2. Strukov DB, Snider GB, Stewart DR, Williams RS. The missing memristor found. Nature. 2008; 453, 80-84. http://dx.doi.org/10.1038/nature06932

3. Tour JM, He T. The fourth element. Nature. 2008;453;42-43. http://dx.doi.org/10.1038/453042a

4. Lütken CA, The missing link in circuit theory. In: Martinsen $\varnothing \mathrm{G}$, Jensen $\varnothing$, An anthology of developments in clinical engineering and bioimpedance. Oslo: Unipub; 2009. p. 17790.

5. Chua LO. Introduction to nonlinear network theory. 1st Ed. New York: McGraw-Hill; 1969.

6. Chua LO, Kang SM. Memristive devices and systems. Proc IEEE. 1976;64;209-23. http://dx.doi.org/10.1109/PROC.1976.10092 
7. Williams RS. How we found the missing memristor. IEEE Spectrum. 2008;45;28-35. http://dx.doi.org/10.1109/MSPEC.2008.4687366

8. Grimnes S, Lütken CA, Martinsen ØG. Memristive properties of electro osmosis in human sweat ducts. WC2009, IFMBE Proceedings. 2009; 25/VII, 696-698. http://dx.doi.org/10.1007/978-3-642-03885-3_193

9. Johnsen GK, Lütken CA, Martinsen ØG, Grimnes S. Memristive model of electro-osmosis in skin. Phys Rev E. 2011;83;031916. http://dx.doi.org/10.1103/PhysRevE.83.031916

10. Pershin YV, Di Ventra M. Memory effects in complex materials and nanoscale systems. Advances in Physics. 2011;60(2);145-227. http://dx.doi.org/10.1080/00018732.2010.544961

11. Chua LO, Nonlinear circuit foundations for nanodevices, Part 1: The four-dimensional torus. Proc IEEE. 2003; 91(11);183059. http://dx.doi.org/10.1109/JPROC.2003.818319

12. Yoglekar YN, Wolf SJ. The elusive memristor: properties of basic electric circuits. Eur J Phys. 2009;30;661-75. http://dx.doi.org/10.1088/0143-0807/30/4/001

13. Cole KS. Rectification and inductance in the squid giant axion. J Gen Physiol. 1941;25;29-51 http://dx.doi.org/10.1085/jgp.25.1.29

14. Cole KS, Membranes, ions, and impulses. University of California Press; Berkeley; 1972.

15. Mauro A. Anomalous impedance, a phenomenological property of time-variant resistance - an analytic review. Biophys J. 1961;1;353-72. http://dx.doi.org/10.1016/S0006-3495(61)86894-X

16. http://www.newscientist.com/article/mg20928024.500-sweatducts-make-skin-a-memristor.html

17. Chua LO, Memristors: A new nanoscale CNN cell. In: Baatar $\mathrm{C}$, et al, editors. Cellular nanoscale sensory wave computing. Boston: Springer; 2010. p. 87-115. http://dx.doi.org/10.1007/978-1-4419-1011-0_4

18. Grimnes S, Martinsen ØG. Bioimpedance and bioelectricity basics. Academic Press; 2008.

19. Malmviou J, Plonsey R. Bioelectromagnetism. Oxford University Press; 1995.

20. Grimnes S. Skin impedance and electro-osmosis in the human epidermis. Med Biol Eng Comp. 1983;21;739-49. http://dx.doi.org/10.1007/BF02464037

21. Licht TS, Stern M, Shwachman H. Measurement of the electrical conductivity of sweat. Clin Chem. 1957;3; 37-48.
22. Grimnes S, Psychogalvanic reflex and changes in electrical parameters of dry skin. Med Biol Eng Comp1982; 20;734-40. http://dx.doi.org/10.1007/BF02442528

23. Tronstad C, Johnsen GK, Grimnes S, Martinsen ØG. A study on electrode gels for skin conductance measurements. Physiol Meas. 2010;31;1395-1410. http://dx.doi.org/10.1088/0967-3334/31/10/008

24. Martinsen ØG, Grimnes S, Karlsen J. Low frequency dielectric dispersion of microporous membranes in electrolyte solution. J Coll Interf Sci. 1998;199;107-10. http://dx.doi.org/10.1006/jcis.1997.5331

25. Hodgkin AL, Huxley AF. A quantitative description of membrane current and its application to conduction in nerve. $\mathrm{J}$ Phys. 1952;117;500-44.

26. Johnston D, Wu SM-S, Biophysics of computation. Oxford; New York; 1994.

27. Dayan P, Abbott LF. Theoretical neuroscience. MIT Press; Cambridge; 2001.

28. Einevoll G. Mathematical modeling of neural activity. In: A. Skjeltorp, editor. Dynamics of Complex Interconnected Systems: Networks and Bioprocesses. NATO Science Series II: Mathematics, Physics and Chemistry. Kluwer Academic; 2006. http://dx.doi.org/10.1007/1-4020-5030-5_8

29. Pershin YV, Di Ventra M. Experimental demonstration of associative memory with memristive neural networks. Neural Networks 2010;23;881-6. http://dx.doi.org/10.1016/j.neunet.2010.05.001

30. Bliss T, Collingridge G, Morris R. LTP - Long term Potenlication. Oxford; New York; 2003.

31. Kandel ER. In search of memory. Norton; New York; 2006.

32. Di Ventra M, Pershin YV, Chua LO. Circuit elements with memory : Memristors, memcapacitors, and meminductors. Proc IEEE. 2009;97;17-17-24.

33. Krems M, Pershin YV, Di Ventra M. Ionic memcapacitive effects in nanopores. Nano Lett. 2011;10;264-78.

34. Prodromakis T, Toumazou C, Chua L. Two centuries of memristors. Nature Materials. 2012; 11;478-81. http://dx.doi.org/10.1038/nmat3338

35. Kavehei O, Iqbal A, Kim YS, Eshragian K, Al-Sarawi SF, Abbott D. The fourth element: characteristics, modelling and electromagnetic theory of the memristor. Proc Royal Soc A Mathem Phys Eng Sci. 2010;466;2175-2202. 